\title{
Effect of the number of parity on right heart chamber quantification
}

\author{
Muhammed Keskin ${ }^{1}$, Edibe Borklu², Selami Doğan ${ }^{1}$, Bayram Ozturk ${ }^{3}$, Adnan Kaya ${ }^{4}$, Lutfi \\ Ocal $^{5}$, Sinan Cersit ${ }^{5}$, Erhan Tenekecioglu ${ }^{6}$, Nurgül Keser ${ }^{1}$, and Ahmet Orhan ${ }^{7}$ \\ ${ }^{1}$ Sultan Abdulhamid Han Egitim ve Arastirma Hastanesi \\ ${ }^{2}$ Istanbul Dr Lütfi Kırdar Kartal Eğitim ve Araştırma Hastanesi \\ ${ }^{3}$ Kahramanmaras Sutcu Imam University \\ ${ }^{4}$ Duzce University \\ ${ }^{5}$ Kartal Kosuyolu Yuksek Ihtisas Egitim ve Arastirma Hastanesi \\ ${ }^{6}$ Bursa City Hospital \\ ${ }^{7}$ Sultan Abdülhamid Han Training and Research Hospital
}

June 11, 2021

\begin{abstract}
Introduction Pregnancy is a process that causes several physiological changes including all systems as well as cardiovascular system. Ventricular hypertrophy and dilation of cardiac chambers are seen as a result of these changes. Although there are studies about pregnancy-related changes in echocardiographic examination; there is no data about the long-term effects of parity on these alterations. Therefore, we have evaluated the long-term effect of pregnancy on right ventricular (RV) dilation and RV hypertrophy and their relation to the parity number. Methods This prospective study included a total of 600 women ( 200 consecutive women who had no parity, 200 women who had a parity number of 1 to 4 and 200 women who had a parity number of more than 4). Right chambers' measurements were compared between the groups. Results In echocardiographic analysis, RV and right atrial dimensions and areas and RV wall thickness were higher in parous women. On the other hand, RV systolic function parameters were significantly lower in parous women. These significant changes showed a gradual increase or decrease by increasing parity number. There were also independent relationship between the number of parity and RV hypertrophy even after adjustment for several confounders. Conclusion Pregnancy-related physiological changes mostly resolve after delivery. This study about long-term effects of pregnancy on RV has demonstrated that there is a significant relation between the number of parity and either RV dilation or RV hypertrophy. Each parity had also additive effect on these changes.
\end{abstract}

\section{Effect of the number of parity on right heart chamber quantification}

Short Title: Parity and the right heart

Keywords : Parity, pregnancy, right heart chambers

Muhammed Keskin, FESC, Assoc. Prof. MD ${ }^{1}$;

Edibe Betul Borklu, $\mathrm{MD}^{2}$; Selami Dogan, $\mathrm{MD}^{1}$;

Bayram Öztürk, $\mathrm{MD}^{3}$; Adnan Kaya, Assoc. Prof. $\mathrm{MD}^{4}$; Lütfi Öcal, $\mathrm{MD}^{5}$;

Sinan Çerşit MD ${ }^{5 ;}$ Erhan Tenekecioğlu, Assoc. Prof. $\mathrm{MD}^{6}$

Nurgül Keser, Prof. MD ${ }^{1}$; Ahmet L. Orhan, Prof. MD ${ }^{1}$; 
1. Cardiology, Health Sciences University, Sultan II. Abdulhamid Han Training and Research Hospital, Istanbul, Turkey.

2. Cardiology, Health Sciences University, Kartal Lutfu Kirdar Training and Research Hospital, Istanbul, Turkey.

3. Cardiology, Sutcu Imam University, Kahramanmaras, Turkey.

4. Cardiology, Duzce University, Duzce, Turkey

5. Cardiology, Kartal Kosuyolu Training and Research Hospital, Istanbul, Turkey.

6. Cardiology, Bursa City Hospital, Bursa, Turkey.

Corresponding Author: Selami DOĞAN, MD

Health Sciences University, Sultan Abdulhamid Han Training and Research Hospital.

Selimiye Neighborhood, Tibbiye Street, 34668, Uskudar, İstanbul, Turkey

Phone: +902165422020 Fax: +90(216) 5422010

E-mail:selamidogan61@gmail.com

Effect of the number of parity on right heart chamber quantification

\section{Abstract}

\section{Introduction}

Pregnancy is a process that causes several physiological changes including all systems as well as cardiovascular system. Ventricular hypertrophy and dilation of cardiac chambers are seen as a result of these changes. Although there are studies about pregnancy-related changes in echocardiographic examination; there is no data about the long-term effects of parity on these alterations. Therefore, we have evaluated the long-term effect of pregnancy on right ventricular (RV) dilation and RV hypertrophy and their relation to the parity number.

\section{Methods}

This prospective study included a total of 600 women (200 consecutive women who had no parity, 200 women who had a parity number of 1 to 4 and 200 women who had a parity number of more than 4 ). Right chambers' measurements were compared between the groups.

\section{Results}

In echocardiographic analysis, RV and right atrial dimensions and areas and RV wall thickness were higher in parous women. On the other hand, RV systolic function parameters were significantly lower in parous women. These significant changes showed a gradual increase or decrease by increasing parity number. By multivariate hierarchical logistic regression analysis, the 4 independent factors that increased the risk of RV dilation were age (OR: 1.16 CI: 1.10 - 1.20), body mass index (OR: 1.05, CI: 1.02 to 1.08), smoking (OR: 1.87, CI: 1.28 to 4.02 ), and giving a birth (OR: 3.94 CI: $1.82-8.81$ ). There were also independent relationship between the number of parity and RV hypertrophy even after adjustment for several confounders.

\section{Conclusion}

Pregnancy-related physiological changes mostly resolve after delivery. This study about long-term effects of pregnancy on RV has demonstrated that there is a significant relation between the number of parity and either RV dilation or RV hypertrophy. Each parity had also additive effect on these changes.

Key words: Pregnacy, parity, right ventricular functions

Effect of the number of parity on right heart chamber quantification

\section{Introduction}


Pregnancy causes several physiological changes as well as changes in cardiovascular system. These changes are usually seen during pregnancy and mostly resolve after delivery. Plasma volume, heart rate and stroke volume increase in pregnancy and because of these changes cardiac output increases (1). The change in stroke volume occurs by means of increase in ventricular muscle mass and cardiac chamber dilation seen in all four cardiac chambers. The effect of stroke volume on cardiac output is more evident in early course of pregnancy and later in the course it is mainly associated with increased heart rate. Besides these changes, left ventricular (LV) ejection fraction and right ventricular (RV) systolic function do not change in pregnancy (2-3). RV has a key role in cardiovascular physiology and shows compensatory changes including RV dilation and hypertrophy in pregnancy (4). Although there are many studies demonstrated the effects of pregnancy on cardiovascular system; the long-term effect of pregnancy on right heart functions has not been studied. We thought that some changes can persist after delivery in some extent and there may be an additive effect of each pregnancy on these changes. Therefore, we have evaluated the long-term effect of pregnancy on RV dilation and RV hypertrophy and their relation of the number of parity.

\section{Methods}

This prospective study was performed in a tertiary center and included a total of 600 consecutive women (200 consecutive women who had no parity, 200 women who had a parity number from 1 to 4 and 200 women who had a parity number more than 4). The minimum time of pregnancy-free interval was 12 months. The duration of study was 4 months, from July 2020 to October 2020. The patients who had no structural heart disease, history of preeclampsia and eclampsia, atrial fibrillation, neither structural nor vascular pulmonary disease, any autoimmune disease and any systemic disorder with the exception of obesity, hypertension (HT), hyperlipidemia (HL) and diabetes mellitus (DM) are included in the present study in order to eliminate the other factors that can cause morphological changes in RV. Ethical clearance was obtained from the ethics and research committee of our university. All participants gave written informed consent before enrollment in the study. The research was conducted in accordance with the principles of the Declaration of Helsinki.

HT was defined as systolic pressure greater than $140 \mathrm{~mm} \mathrm{Hg}$ or diastolic pressure greater than $90 \mathrm{~mm}$ Hg. DM was defined as the use of insulin or antidiabetic agents in the patient's medical history or a fasting glucose level greater than $126 \mathrm{mg} / \mathrm{dl}$. HL was defined as serum total cholesterol $>240 \mathrm{mg} / \mathrm{dl}$, serum triglyceride $>200 \mathrm{mg} / \mathrm{dl}$, low-density lipoprotein cholesterol $>130 \mathrm{mg} / \mathrm{dl}$, and previously diagnosed HL. Echocardiogram was performed using a Vivid 7 system (GE Vingmed Ultrasound AS, Horten, Norway). The parameters assessed were RV length, basal and mid diameters in $\mathrm{cm}$, RV free wall thickness in mm, RV systolic and diastolic areas in $\mathrm{cm}^{2}$, right atrial (RA) area in $\mathrm{cm}^{2}$ and RA volume in $\mathrm{cm}^{3}$, fractional area change (FAC), DTI-derived tricuspid lateral annular systolic velocity wave ( $\left.\mathrm{S}^{\prime}\right)$ in $\mathrm{cm} / \mathrm{s}$, tricuspid annular plane systolic excursion (TAPSE) in $\mathrm{cm}$, estimated pulmonary arterial pressure (PAP) calculated using the tricuspid regurgitation jet, LV end-diastolic dimension and LV ejection fraction. The measurements were done according to American Society of Echocardiography and European Association of Cardiovascular Imaging guidelines (5). RV dilation was defined as RV basal diameter greater than $4,1 \mathrm{~cm}$. RV hypertrophy was defined as RV free wall thickness greater than $5 \mathrm{~mm}$.

\section{Statistical Analysis}

Analyses were performed using Statistical Package for Social Sciences software, version 20.0 (SPSS; IBM, Armonk, New York, USA). Baseline characteristics and echocardiographic parameters were compared among the patients by parity number and categorized; accordingly, nulliparous, 1 to 4 and parity $>4$. KolmogorovSmirnov test was used for testing of normality. Continuous variables were expressed as mean $\pm \mathrm{SD}$ and compared using one-way analysis of variance. Tukey Post-hoc test was performed to reveal the statistical difference between the groups. Continuous variables with skewed distributions compared using the KruskalWallis test and Bonferroni-corrected Mann-Whitney U test was performed to reveal the statistical difference between the groups. Categorical variables were expressed as number and percentages and Pearson's chisquare or Fisher's exact tests were used to evaluate the differences. Hierarchical logistic regression analysis was used for multivariable analysis to evaluate the univariable and multivariable confounders for RV dilation. The odds ratio (OR) indicates the relative risk of RV dilation. Multivariate analysis by stepwise logistic 
regression models (backward elimination) tested variables that were significant at $\mathrm{p}<0.1$ in the univariate analysis. A forward hierarchical logistic regression model was used for multivariable analysis to assess the independent relationship between each parity category and RV dilation and hypertrophy. Two models were generated to obtain the impact of potential confounders on the association between parity category and RV dilation and hypertrophy. These 2 models include: (1) unadjusted; (2) adjusted for age, body mass index, body surface area and smoking. The odds ratio (OR) indicates the relative risk of RV dilation and RV hypertrophy of parity category compared with nulliparity. Intra-observer and interobserver variability were assessed on separate occasions, using new arbitrary images for RV basal dimension and RV thickness blinded to the previous results and shown in Table 4. Fifty subjects were randomly selected from each group for the analyses. For the interobserver variability assessment, the first observer performed the analyses. Second observer repeated the analyses within 24 hours. For assessment of the intra-observer variability the analyses were repeated twice by the first observer within 1 week. Results were analyzed using coefficient of variation where differences between measurements were expressed as the ratio of the standard deviation to the means and multiplied by 100. Statistical significance was defined as a p value $<0.05$.

\section{Results}

The women's baseline characteristics and echocardiographic findings stratified by parity status were listed in Table1. A total of 600 women (mean age $45.1 \pm 6.8$ ) were included. The baseline characteristics of cases were similar in terms of age, hypertension, diabetes mellitus, hyperlipidemia, current smoking status, body mass index and body surface area. In echocardiographic analysis, RV length, mid and basal diameters, RV systolic and diastolic areas, RV wall thickness, RA area and volume, estimated PAP were significantly higher in parous women. On the other hand, parameters using for evaluation of RV function such as TAPSE, FAC, S' velocity were significantly lower in parous women. The decrease in these parameters, however, has not reached under the lower reference limit according to the guideline. Grand multiparous women had the highest values which were higher in parous women and the lowest values which were lower in parous women. LV ejection fraction and LV end-diastolic dimension were similar between the groups. Figure 1 demonstrates the distribution of RV basal diameter stratified by parity category. In nulliparous group, 8 women (4\%) had RV dilation and 6 women (3\%) had RV hypertrophy. In women with a parity number of 1 to 4, 11 women $(5.5 \%)$ had RV dilation and 19 women $(9.5 \%)$ had RV hypertrophy. In grand multiparous group, 42 women $(22.4 \%)$ had RV dilation and 33 women $(16.4 \%)$ had RV hypertrophy.

Two different hierarchical logistic regression analyses were performed and demonstrated in Table 2 and 3. In the first analysis (Table 2), the univariate predictors of RV dilation were age, body mass index, body surfacearea, hyperlipidemia, smokingand giving a birth (being parous vs nulliparous). By multivariate hierarchical logistic regression analysis, the 4 independent factors that increased the risk of RV dilation were age (OR: 1.16 CI: $1.10-1.20$ ), body mass index (OR: 1.05, CI: 1.02 to 1.08), smoking (OR: 1.87, CI: 1.28 to 4.02 ), and giving a birth (OR: $3.94 \mathrm{CI}: 1.82-8.81$ ).

In the second analysis (Table 3) RV dilation and hypertrophy had the highest rates at parity category of $>4$ parity and that had 6.9 and 6.3-times higher than nulliparous category respectively, which had the lowest rates and used as the reference group. This relationship persisted even after adjustment for several confounders; RV dilation and hypertrophy had the highest rates at parity category of $>4$ and that had 5.2 and 5.4-times higher than nulliparous category respectively. Compared with the reference group, the women with the parity category of $1<$ to 4 parity had 3.4 times higher RV hypertrophy and this relationship persisted even after adjustment for several confounders. However, compared with the reference group, the women with the parity category of 1 to 4 parity had higher rates of RV dilation, it did not reach to statistical significance.

\section{Discussion}

We have evaluated the long-term effect of pregnancy on structural and functional echocardiographic measurements of RV and their association with the number of parity. Although the parameters used for evaluation of RV function had never been measured under the lower reference limit in the study population, these 
parameters have reduced in some extent. On the other hand, the structural changes of RV were more evident owing to the findings about RV dilation and RV hypertrophy meaning RV basal diameter and free wall thickness were above the upper reference limit. While the number of parities had a significant effect on these functional and structural changes of RV, giving a birth was found as independent risk factor for RV dilation.

The cardiovascular system undergoes several changes during pregnancy in order to supply metabolic demands for both mother and fetus (6). However, the interesting data demonstrated in our study, was the persistency of some of these physiological changes seen in RV after pregnancy and their relationship with the number of parity. The situation can be explained by cardiac remodeling which is defined as basic changes in geometry, wall thickness, size, and ventricular function by means of complex process including a group of molecular, cellular, and interstitial changes (7-10). Although the exact mechanism of cardiac remodeling is poorly understood, the renin-angiotensin system (RAS) is a key mediator involved in the pathogenesis of cardiac remodeling. Activation of RAS is essential for normal pregnancy in order to be able to cope with the increased demand for salt and water during pregnancy. Many studies have shown that in normal pregnancy there is an increase in almost all the components of the RAS. Therefore, irreversible changes in RV dimensions can be seen by means of remodeling related to activation of RAS in pregnancy (11-13). On the other hand, it should be kept in mind that RAS is activated in pregnancy because of maternal physiological demands and in healthy pregnancy it does not result in pathologic processes such as intrauterine growth restriction, preeclampsia etc. This data also can explain why RV dilation and hypertrophy have found in this study without RV dysfunction which are distinct components of RV remodeling (14-16).

The inflammatory process is also responsible for cardiac remodeling (17). While both pro-inflammatory and anti-inflammatory markers increase in pregnancy, a prospective study comparing the inflammatory mediators between late pregnancy and early postpartum, has showed that markers that have predominantly anti-inflammatory effect were higher in pregnancy (18). In addition, some of the autoimmune diseases such as rheumatoid arthritis can ameliorate during pregnancy in line with this prospective study. On the other hand, most of the physiologic changes resolve during the postpartum period in which pro-inflammatory markers are high. Each pregnancy means exposure of this pro-inflammatory postpartum period that may influence RV remodeling and this situation can be the reason of our finding about significant relation between number of parity and RV dilation and RV hypertrophy. Hormonal alterations have important roles in many physiological changes during pregnancy. Estrogen levels gradually increase and reach highest value in late pregnancy. Eghbali et al demonsrated the association between estrogen level and pregnancy-related heart hypertrophy in pregnant rats (19). Indeed this study is very supportive for our finding about association between RV hypertrophy and parity. Whatever the mechanism of cardiac changes during pregnancy, it is obvious that dilation and hypertrophy of cardiac chambers are compensatory alterations against increased plasma volume and cardiac output. Therefore, these changes are necessary for completion of normal pregnancy and the more pregnancy the woman has, the more she will be exposed to this condition. Thus, association between parity and structural changes of RV is reasonable.

According to our study the other independent risk factors for RV dilation were smoking, age and BMI. Indeed, systemic disease especially HT, DM, smoking, increasing age and obesity have negative effect on cardiac remodeling (20-21). In our study population there were neither resistant HT (HT controlled via [?] 4 antihypertensive drugs) nor complicated DM. This can be the reason why we did not found relation between such diseases and RV dilation. Smoking has structural and functional cardiac effects related to tremendous mechanisms such as hemodynamic and neurohormonal changes, oxidative stress, inflammation etc. Although structural and functional effects of smoking on LV were more studied and well known, in a population-based cohort study, smoking was found independently related to worse RV outcomes (22). From this aspect, our study may give a little contribution to literature even though it was not the main purpose of study. Similarly, obesity has such cardiac alterations either by itself or high incidence of relation with comorbidities such as coronary artery disease, HT, DM. World Health Organization defines overweight and obesity as a BMI over 25 and BMI over 30 respectively. We did not use this classification in study but increasing BMI itself was found as independent risk factor for RV dilation. Again, in line with our study, it has shown in many studies that several structural and functional cardiac alterations are age-related irrespective of comorbidities (23-24). 
Although relation between the number of parity and estimated PAP was found statistically significant, there was not any value exceeding $35 \mathrm{mmHg}$. In fact, transthoracic echocardiography is unreliable method for PAP and overestimates it (25-26). On the other hand, our study may point a linear increase in PAP with increasing number of parity other than causing clinically important outcomes. RA dimensions were also found higher in parous women and it was related to the number of parity. Scarcity of studies about RA and pregnancy makes it difficult to compare, but it can be handled with RV and commentions about RV can be applied to alterations about RA.

Echocardiography is method of choice for assessment of RV. It is widely available and provides useful information about the right heart chambers (27). We have assessed the right heart especially focusing on RV via echocardiography and evaluated the long-term effects of parity on RV and its relation of the number of parities. The studies about the right heart are very few when compared to the left heart and the number of studies declines when it is applied to pregnancy. What makes this study important is its uniqueness indicating the parity had long-term effects on RV and this association has increased in a number-related manner.

\section{Conflict of Interest Disclosures}

None.

\section{Refrences}

1. Osol G, Ko NL, Mandala M. Plasticity of the Maternal Vasculature During Pregnancy. Annual Review of Physiology. 2019;111:81-9.

2. Pillay PS, Catherine NP, Tolppanen H, Mebazaa A. Physiological changes in pregnancy. Cardiovasc J Afr. 2016; 27(2): 89-94.

3. Desai DK, Moodley J, Naidoo DP. Echocardiographic assessment of cardiovascular hemodynamics in normal pregnancy. Obstet Gynecol. 2004; 104:20-29.

4. Kossaify A. Echocardiographic Assessment of the Right Ventricle, from the Conventional Approach to Speckle Tracking and Three-Dimensional Imaging, and Insights into the "Right Way" to Explore the Forgotten Chamber. Clin Med Insights Cardiol.2015;9:65-75.

5. Lang RM, Badano LP, Mor-Avi V et al. Recommendations for cardiac chamber quantification by echocardiography in adults: an update from the American Society of Echocardiography and the European Association of Cardiovascular Imaging. Eur Heart J Cardiovasc Imaging. 2015;16:233-270.

6. Clapp JF, Seaward BL, Sleamaker RH, Hiser J. Maternal physiologic adaptations to early human pregnancy. Am J Obstet Gynecol. 1988;159:1456e1460.

7. Kret M, Arora R. Pathophysiological basis of right ventricular remodeling. J Cardiovasc Pharmacol Ther. 2007;12:5-14.

8. Bartelds B, Borgdorff MAJ, Oosten AS et al. Differential responses of the right ventricle to abnormal loading conditions in mice: pressure vs. volume load. Eur J Heart Fail.2011;13:1275-1282.

9. Borgdorff MAJ, Bartelds B, Dickinson MG, Steendijk P, Vroomen M, Berger RMF. Distinct loading conditions reveal various patterns of right ventricular adaptation. Am J Physiol Heart Circ Physiol. 2013;305:H354-364.

10. Voelkel NF, Quaife RA, Leinwand LA et al. Right Ventricular Function and Failure. Circulation. 2006;114:1883-91.

11. Ellmers LJ, Scott NJA, Medicherla S et al. Transforming Growth Factor- $\beta$ Blockade Down-Regulates the Renin-Angiotensin System and Modifies Cardiac Remodeling after Myocardial Infarction. Endocrinology. 2008;149:5828-34.

12. Xu J, Carretero OA, Liao TD et al. Local angiotensin II aggravates cardiac remodeling in hypertension. Am J Physiol Heart Circ Physiol. 2010;299:1328-38.

13. Irani RA, Xia Y. The Functional Role of the Renin-Angiotensin System in Pregnancy and Preeclampsia. Placenta.2008;29:763-71.

14. Shah DM. Role of the renin-angiotensin system in the pathogenesis of preeclampsia. Am J Physiol Renal Physiol.2005;288:14-25. 
15. Dietz MF, Prihadi EA, Bijl PV et al. Prognostic Implications of Right Ventricular Remodeling and Function in Patients With Significant Secondary Tricuspid Regurgitation. Circulation.2019;140:836845.

16. Lumbers ER, Pringle KG. Roles of the circulating renin-angiotensin-aldosterone system in human pregnancy. Am J Physiol Regul Integr Comp Physiol. 2014; 306:91-101.

17. Sydykov A, Mamazhakypov A, Petrovic A et al. Inflammatory Mediators Drive Adverse Right Ventricular Remodeling and Dysfunction and Serve as Potential Biomarkers. Front Physiol.2018;23(9):609.

18. Brann E, Edvinsson A, Punga AR, Sundström-Poromaa I, Skalkidou A. Inflammatory and antiinflammatory markers in plasma: from late pregnancy to early postpartum. Sci Rep. 2019; 9(1):1863.

19. Eghbali M, Deva R, Alioua A, Minosyan TY, Ruan H, Wang Y, Toro L, Stefani E. Molecular and Functional Signature of Heart Hypertrophy During Pregnancy. Circulation Research. 2005;96(11):120816.

20. Kai H, Kudo H, Takayama N, Yasuoka S, Kajimoto H, Imaizumi T. Large Blood Pressure Variability and Hypertensive Cardiac Remodeling. Circulation Journal. 2009;73:2198.

21. Yap J, Tay WT, Teng TK et al. Association of Diabetes Mellitus on Cardiac Remodeling, Quality of Life, and Clinical Outcomes in Heart Failure With Reduced and Preserved Ejection Fraction. Am J Heart Assoc. 2019; 8:e13114.

22. Mreira HT, Armstrong AC, Nwabuo CC et al. Association of smoking and right ventricular function in middle age: CARDIA study.Open Heart. 2020;7:e001270.

23. Aurigemma GP,Simone G, Fitzgibbons TP. Cardiac Remodeling in Obesity. Circ Cardiovasc Imaging. 2013;6:142-52.

24. Hung CL, Wu YJ, Liu CC et al. Age-related Ventricular Remodeling is an Independent Risk for Heart Failure Symptoms in Subjects With Preserved Systolic Function. Int J Gerontology. 2011;5:17-24.

25. Morton A. Physiological Changes and Cardiovascular Investigations in Pregnancy. Heart Lung Circ. 2021;30:e6-15.

26. Trasca LF, Poenaru E, Patrascu N, Cirstoiu M, Vinereanu D. A comprehensive echocardiographic study of the right ventricular systolic function in pregnant women with inherited thrombophilia. Echocardiography. 2020;37:1037-42.

27. Geva T. MRI Is the Preferred Method for Evaluating Right Ventricular Size and Function in Patients with Congenital Heart Disease. Circ Cardiovasc Imaging.2014;7:190-7.

\section{Legends}

Fig. 1 Box-plot illustrates the distribution of right ventricular basal dimension stratified by parity category. 


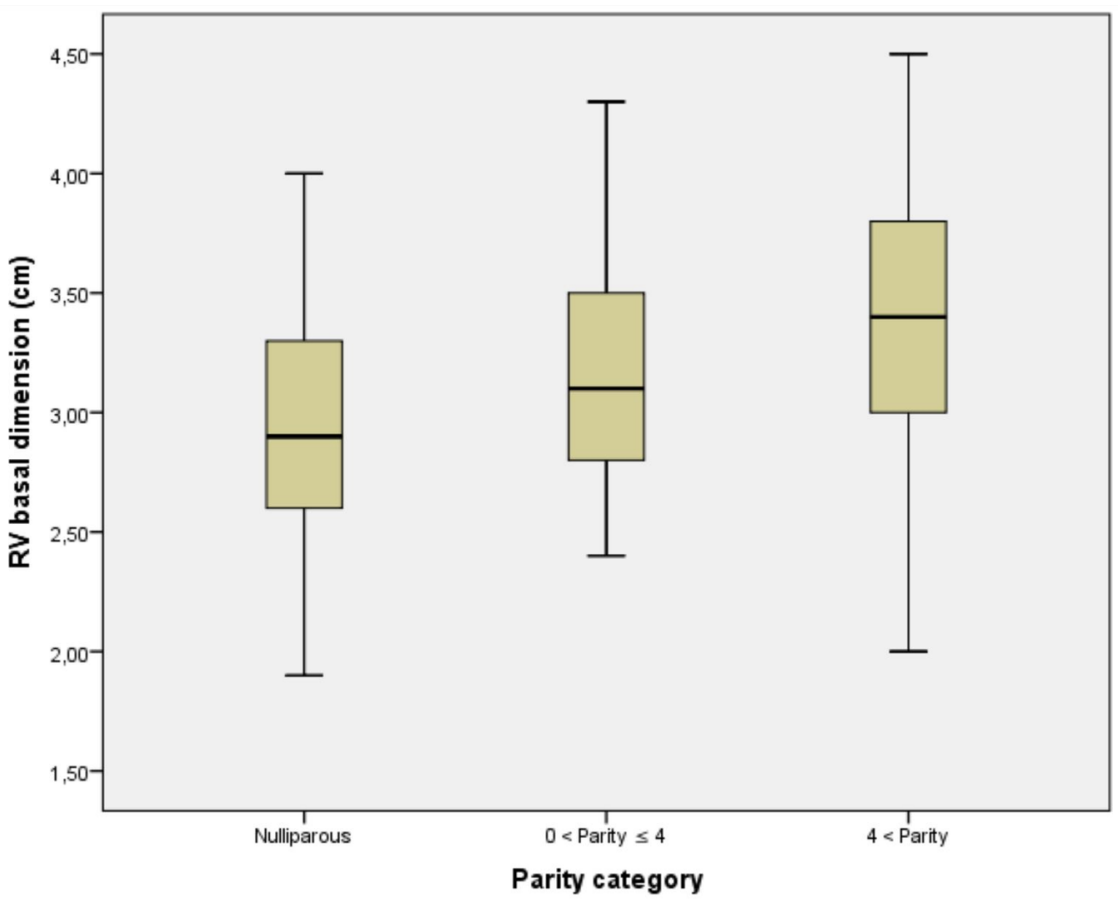

